\title{
THE PROBABILISTIC APPROACH TO THE ANALYSIS OF THE LIMITING BEHAVIOR OF AN INTEGRO-DIFFERENTIAL EQUATION DEPENDING ON A SMALL PARAMETER, AND ITS APPLICATION TO STOCHASTIC PROCESSES ${ }^{1}$
}

\author{
O.V. BORISENKO \\ Kiev Polytechnic Institute \\ Department of Mathematics N3 \\ Prospect Pobedy 3, Kiev-252056, UKRAINE \\ A.D. BORISENKO \\ Kiev University \\ Department of Probability \& Mathematical Statistics \\ Kiev-252017, UKRAINE \\ I.G. MALYSHEV \\ San Jose State University \\ Department of Mathematics \& Computer Science \\ San Jose, CA 95192 USA
}

\begin{abstract}
Using connection between stochastic differential equation with Poisson measure term and its Kolmogorov's equation, we investigate the limiting behavior of the Cauchy problem solution of the integrodifferential equation with coefficients depending on a small parameter. We also study the dependence of the limiting equation on the order of the parameter.
\end{abstract}

Key words: Stochastic process, Kolmogorov's averaging, integro-differential equation, Cauchy problem, limiting behavior, small parameters, white and Poisson noise.

AMS (MOS) subject classifications: $\quad 60 \mathrm{H} 15,60 \mathrm{H} 20,35 \mathrm{R} 60$.

It is well known that investigation of a nonlinear oscillating systems with a small stochastic white noise at the input, can be accomplished applying the averaging method for Kolmogorov's parabolic equation with coefficients depending on a small parameter [1]. If both white and Poisson types of noise are present, then the corresponding Kolmogorov's equation is integro-differential [2],

\footnotetext{
${ }^{1}$ Received: December 1993. Revised: February 1994.
} 
and we shall extend here the averaging principle to such equations.

Let us study behavior, as $\epsilon \rightarrow 0$, of the following equation

$$
\begin{gathered}
\frac{\partial}{\partial t} U(t, x)+\epsilon^{k_{1}}(f(t, x), \nabla U(t, x))+\frac{\epsilon^{k_{2}}}{2} \operatorname{Tr}\left(g(t, x) g^{*}(t, x) \nabla^{2} U(t, x)\right) \\
+\int_{R^{d}}\left[U\left(t, x+\epsilon^{k_{3}} q(t, x, y)\right)-U(t, x)-\epsilon^{k_{3}}(q(t, x, y), \nabla U(t, x))\right] \Pi(d y)=0, \\
(t, x) \in[0, T) \times R^{d},
\end{gathered}
$$

where $\epsilon>0$ is a small parameter and $k_{1}, k_{2}, k_{3}$, are some positive numbers, and

$$
\nabla U(t, x)=\left\{\frac{\partial U(t, x)}{\partial x_{i}}, i=1, \ldots, d\right\}, \nabla^{2} U(t, x)=\left\{\frac{\partial^{2} U(t, x)}{\partial x_{i} \partial x_{j}}, i, j=1, \ldots, d\right\} .
$$

Here $\Pi$ is a finite measure on Borel sets in $R^{d}, f(t, x), q(t, x, y)$ are $d$-dimensional vectors, and $g(t, x)$ is a $d \times d$ square matrix.

\section{Lemma: If}

$$
\lim _{s \rightarrow \infty} \frac{1}{s} \int_{A}^{s+A} b(t, x) d t=\bar{b}(x)
$$

uniformly with respect to $A$ for each $x$, the function $b(x)$ is continuous, and $b(t, x)$ is continuous in $x$ uniformly with respect to $(t, x)$ in arbitrary compact $|x| \leq C$, and stochastic process $\xi(t)$ is continuous, then

$$
\lim _{\epsilon \rightarrow 0} \int_{0}^{t} b\left(\frac{\tau}{\epsilon}, \xi(\tau)\right) d \tau=\int_{0}^{t} \bar{b}(\xi(\tau)) d \tau .
$$

The proof is similar to that in [2].

Now, replacing $t$ with $t / \epsilon^{k}$ in (1), where $k=\min \left(k_{1}, k_{2}, k_{3}\right)$, and denoting $V_{\epsilon}(t, x)=U\left(t / \epsilon^{k}, x\right)$, we can derive the following equation:

$$
\begin{gathered}
\frac{\partial}{\partial t} V_{\epsilon}(t, x)+\epsilon^{k_{1}-k}\left(f\left(t / \epsilon^{k}, x\right), \nabla V_{\epsilon}(t, x)\right)+\frac{\epsilon^{k_{2}-k}}{2} \operatorname{Tr}\left(g\left(t / \epsilon^{k}, x\right) g^{*}\left(t / \epsilon^{k}, x\right) \nabla^{2} V_{\epsilon}(t, x)\right) \\
+\frac{1}{\epsilon^{k}} \int_{R^{d}}\left[V_{\epsilon}\left(t, x+\epsilon^{k_{3}} q\left(t / \epsilon^{k}, x, y\right)\right)-V_{\epsilon}(t, x)-\epsilon^{k_{3}}\left(q\left(t / \epsilon^{k}, x, y\right) \nabla V_{\epsilon}(t, x)\right)\right] \Pi(d y)=0 \\
(t, x) \in[0, T) \times R^{d} .
\end{gathered}
$$


Theorem: Let the following conditions hold:

1) the functions $f(t, x), g(t, x), q(t, x, y)$ are continuous in $(t, x)$, bounded and twice continuously differentiable with respect to $x$, with derivatives also bounded;

2) uniformly with respect to $A$ for each $x \in R^{d}, y \in R^{d}$ there exists the following three limits

and

$$
\lim _{s \rightarrow \infty} \frac{1}{s} \int_{A}^{s+A} f(t, x) d t=\bar{f}(x), \lim _{s \rightarrow \infty} \frac{1}{s} \int_{A}^{s+A} g(t, x) g^{*}(t, x) d t=\bar{G}(x),
$$

$$
\lim _{s \rightarrow \infty} \frac{1}{s} \int_{A}^{s+A} q(t, x, y) q^{*}(t, x, y) d t=\bar{Q}(x, y)
$$

3) The functions $\bar{f}(x), \bar{G}(x), \bar{Q}(x, y)$ satisfy the Lipschitz condition in $x$, and the matrix

is uniformly parabolic.

$$
\bar{B}(x)=\bar{G}(x)+\int_{R^{d}} Q(x, y) \Pi(d y)
$$

Then,

a) if $k_{1}=k_{2}=2 k_{3}$ and $V_{\epsilon}(t, x)$ satisfies (2) and the "Cauchy" condition

$$
\lim _{t \uparrow T} V_{\epsilon}(t, x)=F(x), \quad F(x) \in C_{b}^{2}\left(R^{d}\right),
$$

then $\lim _{\epsilon \rightarrow 0} V_{\epsilon}(t, x)=\bar{V}(t, x)$, where $\bar{V}(t, x)$ is a solution of the problem:

$$
\begin{gathered}
\frac{\partial}{\partial t} \bar{V}(t, x)+(\bar{f}(x), \nabla \bar{V}(t, x))+\frac{1}{2} \operatorname{Tr}\left(\bar{B}(x) \nabla^{2} \bar{V}(t, x)\right)=0, \\
\lim _{t \uparrow T} \bar{V}(t, x)=F(x) .
\end{gathered}
$$

b) If $k<k_{1}$, then $V$ satisfies (4)-(5) but in this case there is no term containing $\bar{f}(x)$ in (4); Similarly, if $k<k_{2}$, then $\bar{B}(x)$ does not depend on $\bar{G}(x)$; and if $k<2 k_{3}$, then $\bar{B}(x)$ does not contain the term

$$
\int_{R^{d}} \bar{Q}(x, y) \Pi(d y)
$$

Proof: Applying the results of [2-3] to the conditions of the theorem, it follows that the solution of the problem (2)-(3) exists for each $\epsilon$, is unique and can be represented in the form 


$$
V_{\epsilon}(t, x)=E\left[F\left(\xi_{\epsilon}(t, x, T)\right)\right],
$$

where $\xi_{\epsilon}(t, x, T)$ is the solution of the stochastic equation

$$
\begin{gathered}
\xi_{\epsilon}(t, x, s)=x+\epsilon^{k_{1}-k} \int_{t}^{s} f\left(\tau / \epsilon^{k}, \xi_{\epsilon}(t, x, \tau)\right) d \tau \\
+\epsilon^{\frac{1}{2}\left(k_{2}-k\right)} \int_{t}^{s} g\left(\tau / \epsilon^{k}, \xi_{\epsilon}(t, x, \tau)\right) d w(\tau) \\
+\epsilon^{k_{3}} \int_{t}^{s} \int_{R^{d}} q\left(\tau / \epsilon^{k}, \xi_{\epsilon}(t, x, \tau), y\right) \tilde{\nu}(d \tau, d y)
\end{gathered}
$$

where $w(t)$ is a $d$-dimensional Wiener process, $\nu(t, A)$ is a Poisson measure independent of $w, A$ is a Borel set of $R^{d}$, and

$$
\widetilde{\nu}(t, A)=\nu\left(t / \epsilon^{k}, A\right)-t \Pi(A) / \epsilon^{k} ; \quad E \nu(t, A)=t \Pi(A) .
$$

Let

$$
\begin{gathered}
\zeta_{\epsilon}(t, x, s)=\epsilon^{\frac{1}{2}\left(k_{2}-k\right)} \int_{t}^{s} g\left(\tau / \epsilon^{k}, \xi_{\epsilon}(t, x, \tau)\right) d w(\tau) \\
+\epsilon^{k_{3}} \int_{t}^{s} \int_{R^{d}} q\left(\tau / \epsilon^{k}, \xi_{\epsilon}(t, x, \tau), y\right) \tilde{\nu}_{\epsilon}(d \tau, d y) .
\end{gathered}
$$

Then we can obtain the following estimates:

$$
\begin{aligned}
& E\left|\xi_{\epsilon}(t, x, s)\right|^{2} \leq C\left[x^{2}+\left(\epsilon^{2\left(k_{1}-k\right)}+\epsilon^{k_{2}-k}+\epsilon^{2 k_{3}-k}\right)|s-t|\right], \\
& E\left|\zeta_{\epsilon}(t, x, s)\right|^{2} \leq C\left(\epsilon^{k_{2}-k}+\epsilon^{2 k_{3}-k}\right)|s-t|, \\
& E\left|\xi_{\epsilon}\left(t, x, s_{2}\right)-\xi_{\epsilon}\left(t, x, s_{1}\right)\right|^{2} \leq C\left[\epsilon^{2\left(k_{1}-k\right)}\left|s_{2}-s_{1}\right|^{2}+\left(\epsilon^{k_{2}-k}+\epsilon^{2 k_{3}-k}\right)\left|s_{2}-s_{1}\right|\right], \\
& E\left|\zeta_{\epsilon}\left(t, x, s_{2}\right)-\zeta_{\epsilon}\left(t, x, s_{1}\right)\right|^{2} \leq C\left(\epsilon^{k_{2}-k}+\epsilon^{2 k_{3}-k}\right)\left|s_{2}-s_{1}\right| .
\end{aligned}
$$

From these estimates we infer that the family of processes $\left(\xi_{\epsilon}(t, x, s), \zeta_{\epsilon}(t, x, s)\right)$ satisfies the Skorokhod's compactness conditions [4]. Therefore, for any sequence $\epsilon \rightarrow 0$ there exists a subsequence $\epsilon_{m} \rightarrow 0, m=1,2, \ldots$, and processes $\bar{\xi}(t, x, s)$, $\bar{\zeta}(t, x, s)$ such that $\xi_{\epsilon_{m}}(t, x, s) \rightarrow \xi(t, x, s), \quad \zeta_{\epsilon_{m}}(t, x, s) \rightarrow \zeta(t, x, s)$ in probability as $\epsilon_{m} \rightarrow 0$. From (6) we can also find that

$$
\xi_{\epsilon_{m}}(t, x, s)=x+\epsilon_{m}^{k_{1}-k} \int_{t}^{s} f\left(\tau / \epsilon_{m}^{k}, \xi_{\epsilon_{m}}(t, x, \tau)\right) d \tau+\zeta_{\epsilon_{m}}(t, x, s) .
$$

Then, for any fixed $(t, x) \in[0, T]$ we have: 


$$
\begin{gathered}
E\left|\xi_{\epsilon}\left(t, x, s_{2}\right)-\xi_{\epsilon}\left(t, x, s_{1}\right)\right|^{4} \leq C\left[\epsilon^{4\left(k_{1}-k\right)}\left|s_{2}-s_{1}\right|^{4}+E\left|\zeta_{\epsilon}\left(t, x, s_{2}\right)-\zeta_{\epsilon}\left(t, x, s_{1}\right)\right|^{4}\right] \\
E\left|\zeta_{\epsilon}\left(t, x, s_{2}\right)-\zeta_{\epsilon}\left(t, x, s_{1}\right)\right|^{4} \leq C\left[\left(\epsilon^{2\left(k_{2}-k\right)}+\epsilon^{2\left(2 k_{3}-k\right)}\right)\left|s_{2}-s_{1}\right|^{2}\right. \\
\left.+\epsilon^{4 k_{3}-3 k / 2}\left|s_{2}-s_{1}\right|^{3 / 2}+\epsilon^{4 k_{3}-k}\left|s_{2}-s_{1}\right|\right] .
\end{gathered}
$$

Therefore,

$$
\begin{gathered}
E\left|\bar{\xi}\left(t, x, s_{2}\right)-\bar{\xi}\left(t, x, s_{1}\right)\right|^{4} \leq C\left[\left|s_{2}-s_{1}\right|^{4}+\left|s_{2}-s_{1}\right|^{2}\right] \\
E\left|\bar{\zeta}\left(t, x, s_{2}\right)-\bar{\zeta}\left(t, x, s_{1}\right)\right|^{4} \leq C\left|s_{2}-s_{1}\right|^{2}
\end{gathered}
$$

and the processes $\bar{\xi}(t, x, s), \bar{\zeta}(t, x, s)$ satisfy the Kolmogorov's continuity condition on $s[5]$.

a) Let us consider the case $k_{1}=k_{2}=2 k_{3}$. Then from (7) we obtain:

$$
\xi_{\epsilon}(t, x, s)=x+\int_{t}^{s} f\left(\tau / \epsilon^{k}, \xi_{\epsilon}(t, x, \tau)\right) d \tau+\zeta_{\epsilon}(t, x, s)
$$

From this point we shall omit the subindex $m$ in $\epsilon_{m}$ for simplicity. Then for each fixed $(t, x) \in[0, T]$ the process

$$
\zeta_{\epsilon}(t, x, s)=\int_{t}^{s} g\left(\tau / \epsilon^{k}, \xi_{\epsilon}(t, x, \tau)\right) d w(\tau)+\epsilon^{k / 2} \int_{t}^{s} \int_{R^{d}} q\left(\tau / \epsilon^{k}, \xi_{\epsilon}(t, x, \tau), y\right) \tilde{\nu}(d \tau, d y)
$$

is a vector-valued martingale with matrix characteristic

$$
\begin{gathered}
\left\langle\zeta_{\epsilon}(t, x, s), \zeta_{\epsilon}(t, x, s)\right\rangle=\int_{t}^{s} g\left(\tau / \epsilon^{k}, \xi_{\epsilon}(t, x, \tau)\right) g^{*}\left(\tau / \epsilon^{k}, \xi_{\epsilon}(t, x, \tau)\right) d \tau \\
+\int_{t}^{s} \int_{R^{d}} q\left(\tau / \epsilon^{k}, \xi_{\epsilon}(t, x, \tau), y\right) q^{*}\left(\tau / \epsilon^{k}, \xi_{\epsilon}(t, x, \tau), y\right) \Pi(d y) d \tau
\end{gathered}
$$

Using the above lemma, it is easy to show that

and

$$
P-\lim _{\epsilon \rightarrow 0} \int_{t}^{s} f\left(\tau / \epsilon^{k}, \xi_{\epsilon}(t, x, \tau)\right) d \tau=\int_{t}^{s} \bar{f}(\bar{\zeta}(t, x, \tau)) d \tau
$$

$$
P-\lim _{\epsilon \rightarrow 0}\left\langle\zeta_{\epsilon}(t, x, s), \zeta_{\epsilon}(t, x, s)\right\rangle=\int_{t}^{s} \bar{B}(\bar{\zeta}(t, x, \tau)) d \tau
$$

Hence, from (8), (9), and (10) we obtain a continuous square integrable vectorvalued martingale

with matrix characteristic

$$
\bar{\zeta}(t, x, s)=x+\int_{t}^{s} \bar{f}(\bar{\zeta}(t, x, \tau)) d \tau+\bar{\zeta}(t, x, s)
$$




$$
\langle\bar{\zeta}(t, x, s), \bar{\zeta}(t, x, s)\rangle=\int_{t}^{s} \bar{B}(\bar{\zeta}(t, x, \tau)) d \tau .
$$

It follows from [6] that there exists a $d$-dimensional Wiener process $w(t)$ such that

where

$$
\bar{\zeta}(t, x, s)=\int_{t}^{s} \bar{\sigma}(\bar{\zeta}(t, x, \tau)) d \bar{w}(\tau)
$$

$$
\bar{\sigma}(x) \bar{\sigma}^{*}(x)=\bar{B}(x)
$$

Consequently, the process $\bar{\xi}(t, x, s)$ satisfies the equation which, according [2], has a unique solution:

$$
\bar{\xi}(t, x, s)=x+\int_{t}^{s} \bar{f}(\bar{\xi}(t, x, \tau)) d \tau+\int_{t}^{s} \bar{\sigma}(\bar{\xi}(t, x, \tau)) d \bar{w}(\tau)
$$

The matrix $\bar{B}(x)$ is positive definite for all $x \in R^{d}$, satisfies Lipschitz conditions, and therefore matrix $\bar{\sigma}(x)$ satisfies Lipschitz condition as well. Then, using the Lebesgue dominated convergence theorem, we obtain

$$
\lim _{\epsilon_{m} \rightarrow 0} V_{\epsilon_{m}}(t, x)=\bar{V}(t, x)=E[F(\bar{\xi}(t, x, T))]
$$

for any sequence $\epsilon_{m} \rightarrow 0$. But as it follows from [7] the function $\bar{V}(t, x)$ is a unique solution of the problem (4)-(5), which completes the proof of the part $a$ ) of the theorem.

b) When $k<k_{1}$, the boundedness of $f(t, x)$ implies that

$$
E\left|\int_{t}^{s} f\left(\tau / \epsilon^{k}, \xi_{\epsilon}(t, x, \tau)\right) d \tau\right| \leq C
$$

and therefore the second term in the right side of (6) converges to 0 with $\epsilon \rightarrow 0$ in probability. The matrix characteristic of the martingale $\zeta_{\epsilon}(t, x, s)$ in $(7)$ has the form

$$
\begin{gathered}
\left\langle\zeta_{\epsilon}, \zeta_{\epsilon}\right\rangle=\epsilon^{k_{2}-k} \int_{t}^{s} g\left(\tau / \epsilon^{k}, \xi_{\epsilon}(t, x, \tau)\right) g^{*}\left(\tau / \epsilon^{k}, \xi_{\epsilon}(t, x, \tau)\right) d \tau \\
+\epsilon^{2 k_{3}-k} \int_{t}^{s} \int_{R^{d}} q\left(\tau / \epsilon^{k}, \xi_{\epsilon}(t, x, \tau), y\right) q^{*}\left(\tau / \epsilon^{k}, \xi_{\epsilon}(t, x, \tau), y\right) \Pi(d y) d \tau .
\end{gathered}
$$

From the boundedness of $g, q$, similarly to the inference made above, we obtain 
that either first or second term in the right side of (12) converges to 0 (respectively to the $k<k_{2}$ or $k<2 k_{3}$ case) as $\epsilon \rightarrow 0$, which allows to complete the proof of the theorem as in part $a$ ).

\section{REFERENCES}

[1] Mitropolsky, Yu. A., Averaging Method in Nonlinear Mechanics, Naukova Dumka, Kiev 1971.

[2] Gikhman, I.I., Skorokhod, A.V., Stochastic Differential Equations, Springer-Verlag, Berlin 1972.

[3] Borisenko, A.D., The continuous dependence on parameter of the solution of an integrodifferential equation, Teor. Veroyatnost. i Mat. Statist. Vyp. 49 (1993) - to appear.

[4] Skorokhod, A.V., Studies in the Theory of Random Processes, Addison-Wesley 1965.

[5] Gikhman, I.I., Skorokhod, A.V., The Theory of Stochastic Processes, v. 1, SpringerVerlag, Berlin 1974.

[6] Ikeda, N., Watanabe, S., Stochastic Differential Equations and Diffusion Processes, North Holland, Amsterdam and Kodansha, Tokyo 1981.

[7] Friedman, A., Stochastic Differential Equations and Applications, v. 1, Academic Press, New York 1975. 


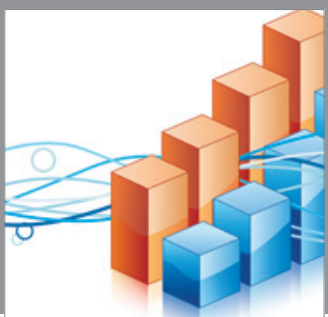

Advances in

Operations Research

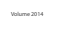

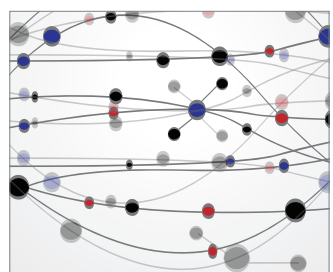

\section{The Scientific} World Journal
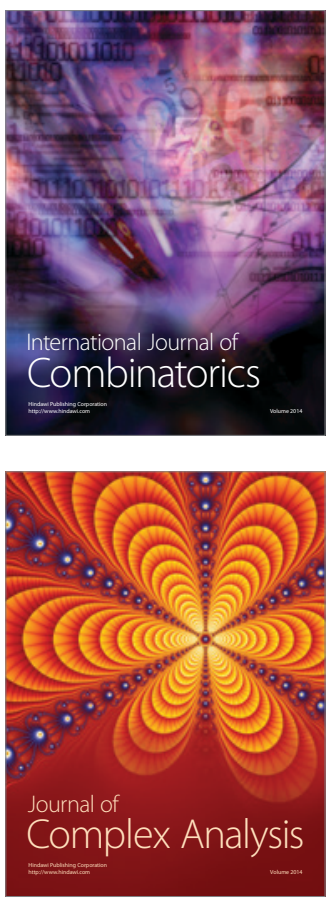

International Journal of

Mathematics and

Mathematical

Sciences
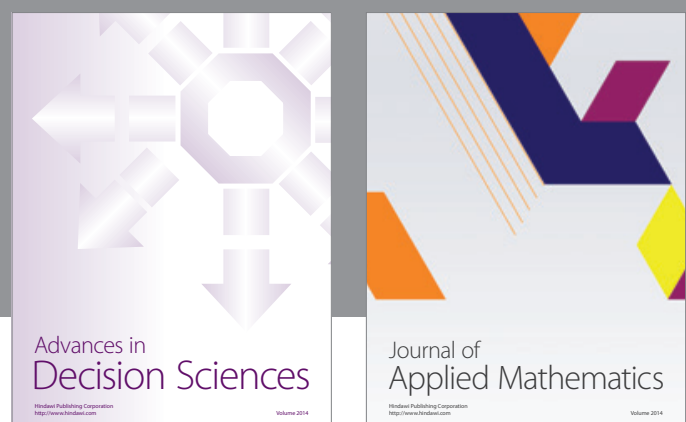

Journal of

Applied Mathematics
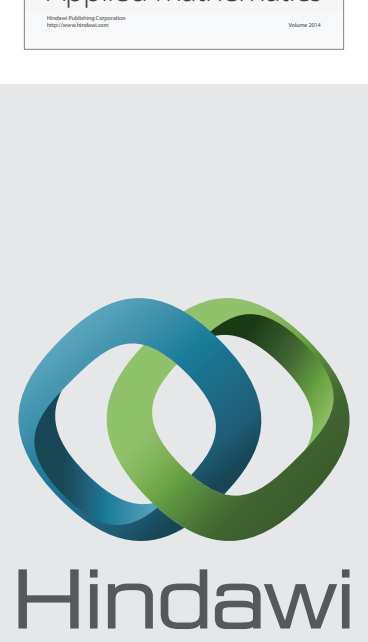

Submit your manuscripts at http://www.hindawi.com
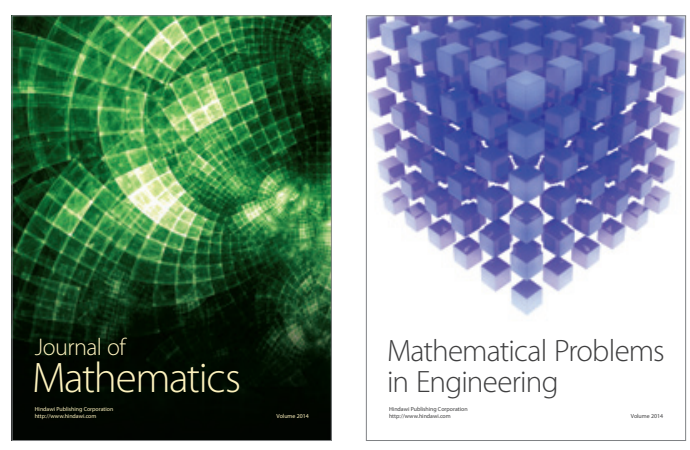

Mathematical Problems in Engineering
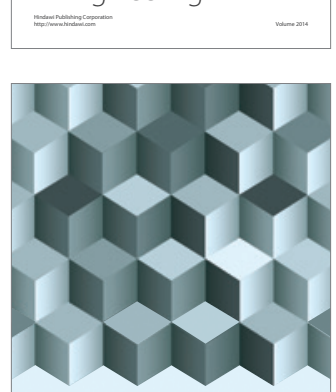

Journal of

Function Spaces
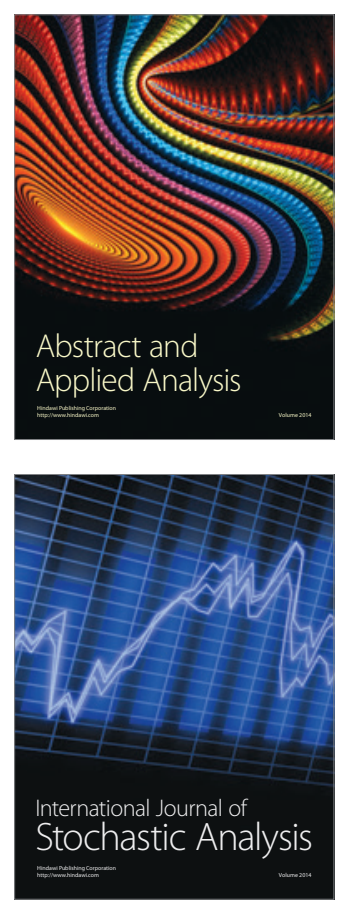

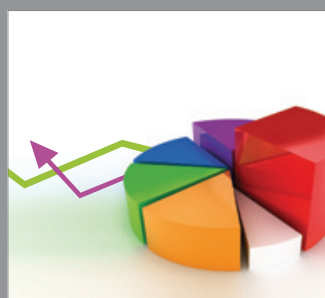

ournal of

Probability and Statistics

Promensencen
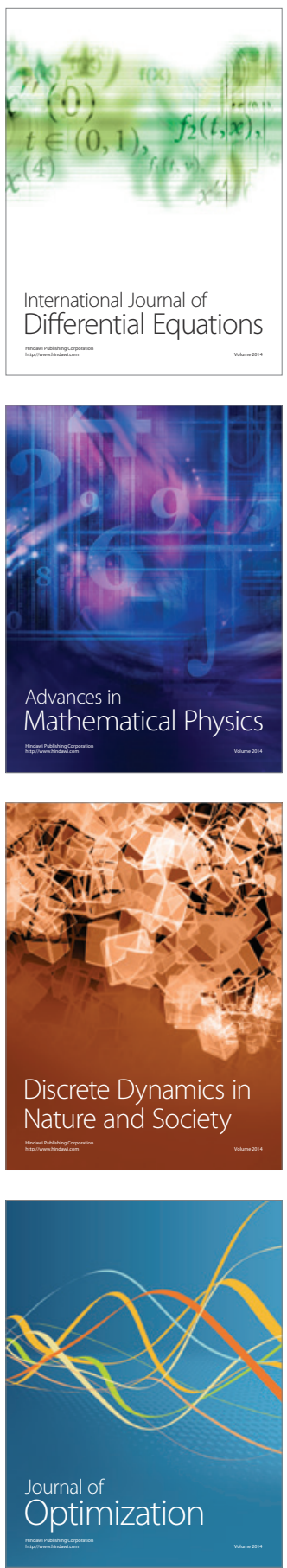